\title{
The conjugacy class graphs of non-abelian 3-groups
}

\author{
Athirah Zulkarnain, Nor Haniza Sarmin, Hazzirah Izzati Mat Hassim* \\ Department of Mathematical Sciences, Faculty of Science, Universiti Teknologi Malaysia, 81310 UTM Johor Bahru, Johor, Malaysia
}

*Corresponding author: hazzirah@utm.my

\section{Article history}

Received 27 February 2019

Revised 31 Mac 2019

Accepted 31 October 2019

Published Online 15 June 2020

\begin{abstract}
A graph is formed by a pair of vertices and edges. It can be related to groups by using the groups properties for its vertices and edges. The set of vertices of the graph comprises the elements or sets from the group while the set of edges of the graph is the properties and condition for the graph. A conjugacy class of an element $x$ is the set of elements that are conjugated with $x$. Any element of a group $G$, labelled as $g$, is conjugated to $x$ if it satisfies $a g a^{-1}=x$ for some elements $a$ in $G$ with its inverse $a^{-1}$. A conjugacy class graph of a group $G$ is defined when its vertex set is the set noncentral conjugacy classes of $G$. Two distinct vertices $A$ and $B$ are connected by an edge if and only if their cardinalities are not co-prime, which means that the order of the conjugacy classes of $A$ and $B$ have common factors. Meanwhile, a simple graph is the graph that contains no loop and no multiple edges. A complete graph is a simple graph in which every pair of distinct vertices is adjacent. Moreover, a $p$-group is the group with prime power order. In this paper, the conjugacy class graphs for some non-abelian 3-groups are determined by using the group's presentations and the definition of conjugacy class graph. There are two classifications of the non-abelian 3-groups which are used in this research. In addition, some properties of the conjugacy class graph such as the chromatic number, the dominating number, and the diameter are computed. A chromatic number is the minimum number of vertices that have the same colours where the adjacent vertices have distinct colours. Besides, a dominating number is the minimum number of vertices that is required to connect all the vertices while a diameter is the longest path between any two vertices. As a result of this research, the conjugacy class graphs of these groups are found to be complete graphs with chromatic number, dominating number and diameter that are equal to eight, one and one, respectively.
\end{abstract}

Key words: Conjugacy class graph, $p$-group, chromatic number, dominating number, diameter

\section{INTRODUCTION}

The conjugacy class graph has been introduced by Bertram et al. in 1990 [1] and many researchers are interested in studying the relation of conjugacy class graph with groups. Casolo and Dolfi [2] experimented on the diameter of conjugacy class graph for finite groups in 1996. In 2013, Moradipour et al. [3] investigated the conjugacy class graph of some metacyclic 2-groups where the chromatic number and the clique number of these graphs are found to be similar. Meanwhile, in the same year, Omar et al. [4] constructed the conjugacy class graph using the probability of metacyclic 2-groups. Later, Omar et al. [5] explored the conjugacy class graphs for the probability of symmetic groups. In this research, the conjugacy class graphs on non-abelian 3-groups [6] are constructed based on two classifications of these groups, which are:

$$
\begin{aligned}
& G=<x, y: x^{3}=y^{3}=z^{3}=1, z=[x, y], z x=x z, z y=y z> \\
& H=<x, y: x^{9}=y^{3}=1, y x y^{-1}=x^{4}>
\end{aligned}
$$

\section{Basic definitions in group theory}

Some basic definitions in group theory used in this research such as the center of a group, the conjugate element, and the conjugacy class of an element of a group, are given in this section.
Definition 1 [7] The center $Z(G)$ of a group $G$ is the set of elements that commute with all elements of $G$ where $Z(G)=\{a \in G: a g=g a$, $\forall g \in G\}$.

Definition 2 [1] If $G$ is a group and $a \in G$, then the conjugate of $a$ is any element in $G$ of the form $\mathrm{gag}^{-1}$ where $g \in G$.

Definition 3 [8] Let $a, b \in G$. We say $a$ and $b$ are conjugated in $G$ or $b$ is a conjugate of $a$ if $x^{-1} a x=b$ for some $x$ in $G$. The conjugacy class of $a$ is the set $\operatorname{cl}(a)=\left\{x^{-1} a x \mid x \in G\right\}$.

\section{Basic definitions in graph theory}

Some basic definitions in graph theory are used in this research including the conjugacy class graph, the complete graph, the chromatic number, the dominating number and the diameter of a graph, are given in this section.

Definition 4 [9] Let $G$ be a group and $Z(G)$ is the center of $G$. A conjugacy class graph of $G$ is a graph when its vertices are the noncentral conjugacy classes of $G$, and two distinct vertices are connected if their cardinalities are not co-prime. A conjugacy class graph of $G$ is denoted as $\Gamma_{G}^{C C}$. 
Definition 5 [10] A complete graph is a simple graph in which every pair of distinct vertices is adjacent. The complete graph with $n$ vertices is denoted as $K_{n}$.

Definition 6 [11] A chromatic number of a graph $\Gamma_{G}$, denoted as $\chi\left(\Gamma_{G}\right)$, is a proper colouring or simply a colouring of the vertices of $G$ in such a way that adjacent vertices have distinct colours. It is also the minimal number of colours in a (vertex) colouring of $G$.

Definition 7 [12] A set $S$ of vertices of $G$ is the dominating set of $G$ if every vertex in $V(G)-S$ is adjacent to some vertices in $S$. A dominating number of $G$, denoted as $\gamma\left(\Gamma_{G}\right)$, is the cardinality of a minimum dominating set.

Definition 8 [10] The diameter of a connected graph $\Gamma$, denoted as diam $(\Gamma)$ with $n$ vertices, is the maximum possible distance between any two vertices.

\section{RESULTS AND DISCUSSION}

This section presents the results of the conjugacy class graphs for nonabelian 3-groups of order 27 along with some properties of the graphs, given in the following theorems and propositions.

\section{The conjugacy class graphs for $G$ and their properties}

In this section, the results for the conjugacy class graph of $G$ are shown as in the following theorem and propositions.

Theorem 1 Let $G=<x, y: x^{3}=y^{3}=z^{3}=1, z=[x, y], z x=$ $x z, z y=y z>$ be a non-abelian 3-group of order 27. Then, the conjugacy class graph of $G$ is the complete graph with eight vertices, namely as $\Gamma_{G}^{C C}=K_{8}$.

Proof The elements of $G$ are as in the following:

$$
\begin{aligned}
& G=\left\{1, x^{2} y x y^{2}, x^{2} y^{2} x y, x, x^{2}, y, x y, x^{2} y, y x, x y x, x^{2} y x, y^{2}, x y^{2},\right. \\
& x^{2} y^{2}, y x^{2}, x y x^{2}, x^{2} y x^{2}, y x y, x y x y, x^{2} y x y, y^{2} x, x y^{2} x, x^{2} y^{2} x, \\
&\left.y x y^{2}, x y x y^{2}, y^{2} x y, x y^{2} x y\right\} .
\end{aligned}
$$

By using Definition 2, the set of conjugate elements of $G$ is obtained as in the following:

1. $\quad c l(1)=\{1\}$

2. $\quad c l\left(x^{2} y x y^{2}\right)=\left\{x^{2} y x y^{2}\right\}$

3. $\quad c l\left(x^{2} y^{2} x y\right)=\left\{x^{2} y^{2} x y\right\}$

4. $\quad c l(x)=\left\{x, y x y^{2}, y^{2} x y\right\}$

5. $\quad \operatorname{cl}\left(x^{2}\right)=\left\{x^{2}, x y x y^{2}, x y^{2} x y\right\}$

6. $\quad c l(y)=\left\{y, x^{2} y x, x y x^{2}\right\}$

7. $\quad \operatorname{cl}(x y)=\left\{x y, y x, x^{2} y x^{2}\right\}$

8. $\quad c l\left(x^{2} y\right)=\left\{x^{2} y, x y x, y x^{2}\right\}$

9. $\quad \operatorname{cl}\left(y^{2}\right)=\left\{y^{2}, x^{2} y x y, x^{2} y^{2} x\right\}$

10. $\operatorname{cl}\left(x y^{2}\right)=\left\{x y^{2}, y x y, y^{2} x\right\}$

11. $\operatorname{cl}\left(x^{2} y^{2}\right)=\left\{x^{2} y^{2}, x y x y, x y^{2} x\right\}$

From the set of conjugate elements, the centers - of $G$ is $Z(G)=$ $\left\{1, x^{2} y x y^{2}, x^{2} y^{2} x y\right\}$. Then, by using Definition 3, the cardinalities of the non-central conjugacy classes of $G$ are as in the following:

$\begin{array}{ll}\text { 1. } & |\operatorname{cl}(x)|=3 \\ \text { 2. } & \left|\operatorname{cl}\left(x^{2}\right)\right|=3 \\ \text { 3. } & |\operatorname{cl}(y)|=3 \\ \text { 4. } & |\operatorname{cl}(x y)|=3 \\ \text { 5. } & \left|\operatorname{cl}\left(x^{2} y\right)\right|=3 \\ \text { 6. } & \left|\operatorname{cl}\left(y^{2}\right)\right|=3 \\ \text { 7. } & \left|\operatorname{cl}\left(x y^{2}\right)\right|=3 \\ \text { 8. } & \left|\operatorname{cl}\left(x^{2} y^{2}\right)\right|=3\end{array}$

The set of non-central conjugacy classes of $G$, which is the set of vertices of the conjugacy class graph of $G$ that have the same cardinalities, which is three. Then, all the vertices are connected with each other and a complete graph with eight vertices has been formed as illustrated in the following Fig. 1.

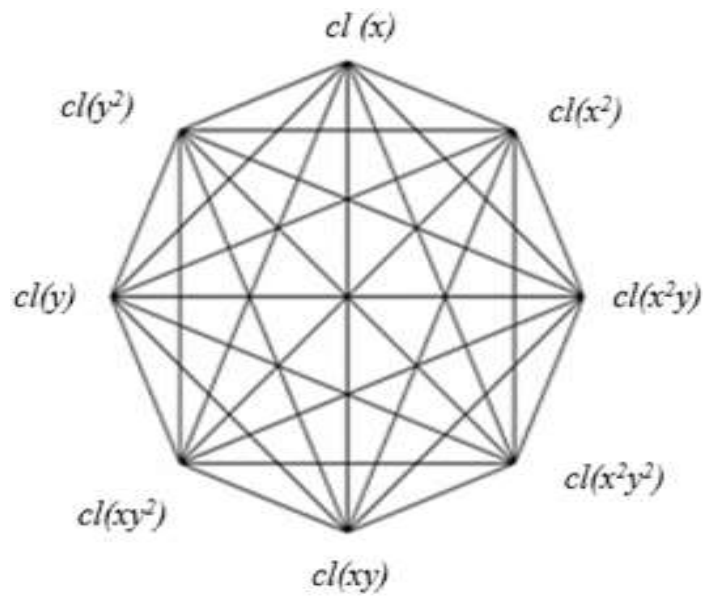

Fig. 1 The conjugacy class graph of $G$.

Proposition 1 Let $G=\left\langle x, y: x^{3}=y^{3}=z^{3}=1, z=[x, y], z x=\right.$ $x z, z y=y z>$ be a non-abelian 3-group of order 27. Then, the chromatic number of the commuting graph of $G$ is eight, namely as $\chi\left(\Gamma_{G}^{C C}\right)=8$.

Proof By referring to the Fig. 1, the minimum number of colours needed to color all the vertices in a way that all the adjacent vertices have different colours is eight. Then, by using Definition 6 , the chromatic number of the conjugacy class graph of $G$ is eight as illustrated in the Fig. 2.

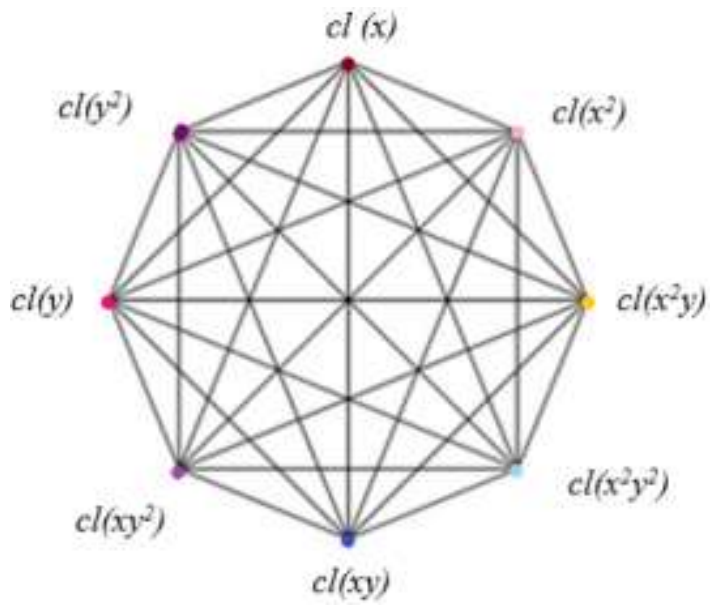

Fig. 2 The colouring of the conjugacy class graph of $G$.

Proposition 2 Let $G=\left\langle x, y: x^{3}=y^{3}=z^{3}=1, z=[x, y], z x=\right.$ $x z, z y=y z>$ be a non-abelian 3-group of order 27. Then, the dominating number of the conjugacy class graph of $G$ is one, namely as $\gamma\left(\Gamma_{G}^{C C}\right)=1$.

Proof By referring to Figure 1, the minimum number of vertices needed to ensure all the vertices are adjacent is one. Then, by using Definition 7, the dominating number of the conjugacy class graph of $G$ is one.

Proposition 3 Let $G=<x, y: x^{3}=y^{3}=z^{3}=1, z=[x, y], z x=$ $x z, z y=y z>$ be a non-abelian 3-group of order 27. Then, the diameter of the conjugacy class graph of $G$ is one, namely as $\operatorname{diam}\left(\Gamma_{G}^{C C}\right)=1$. 
Proof By referring to Fig. 1, the longest path between any two distinct vertices is one. By using Definition 8 , the diameter of conjugacy class graph of $G$ is one.

By using similar method, the results for $H$ are obtained as stated in the following theorem and propositions.

\section{The conjugacy class graph and its properties of the graph for $H$}

This section is on the results for the conjugacy class graph and its properties for the graph of $H$. The proofs are similar as in the previous section.

Theorem 2 Let $H=<x, y: x^{9}=y^{3}=1, y x y^{-1}=x^{4}>$ be a nonabelian 3-group of order 27. Then, the conjugacy class graph of $H$ is the complete graph with eight vertices, namely $\Gamma_{H}^{C C}=K_{8}$.

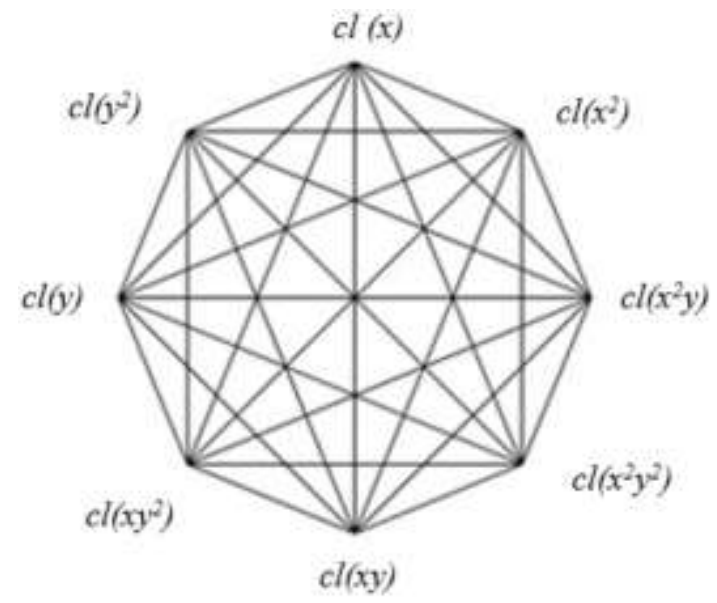

Fig. 3 The conjugacy class graph of $H$.

Proposition 4 Let $H=<x, y: x^{9}=y^{3}=1, y x y^{-1}=x^{4}>$ be a nonabelian 3-group of order 27. Then, the chromatic number of the commuting graph of $H$ is eight, namely $\chi\left(\Gamma_{H}^{C C}\right)=8$.

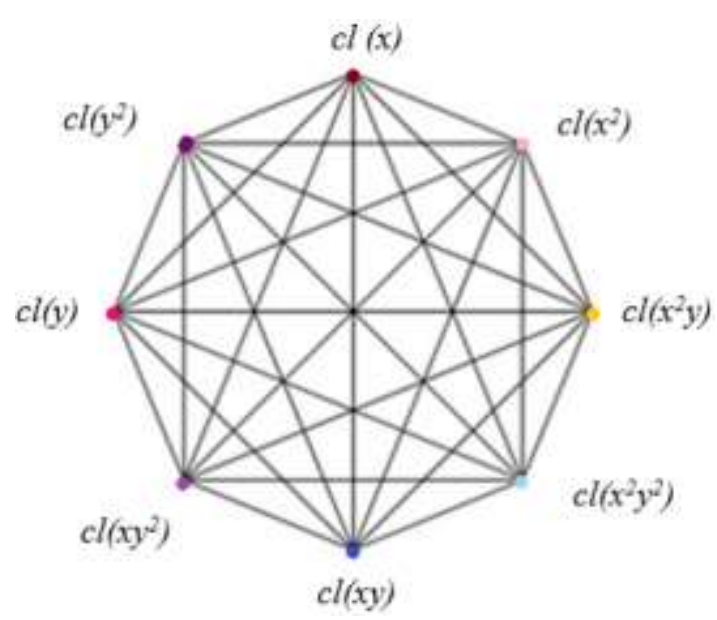

Fig. 4 The colouring of the conjugacy class graph of $H$.

Proposition 5 Let $H=<x, y: x^{9}=y^{3}=1, y x y^{-1}=x^{4}>$ be a nonabelian 3-group of order 27. Then, the dominating number of the conjugacy class graph of $H$ is one, namely $\gamma\left(\Gamma_{H}^{C C}\right)=1$.

Proposition 6 Let $H=<x, y: x^{9}=y^{3}=1, y x y^{-1}=x^{4}>$ be a nonabelian 3-group of order 27. Then, the diameter of the conjugacy class graph of $H$ is one, namely $\operatorname{diam}\left(\Gamma_{H}^{C C}\right)=1$.

\section{CONCLUSION}

The conjugacy class graphs of $G$ and $H$ are turned out to be complete graphs with eight vertices. In addition, the properties of the conjugacy class graphs for $G$ and $H$, which are the diameter is found to be one, the chromatic number is found to be eight, and the dominating number is found to be one.

\section{ACKNOWLEDGEMENT}

This work was financially supported by Ministry of Higher Education Malaysia and Universiti Teknologi Malaysia under UTM Zamalah Scholarship.

\section{REFERENCES}

[1] Bertram, E. A., Herzog, M., Mann, A. On a graph related to conjugacy classes of groups. Bulletin of the London Mathematical Society, 1990. 22(6):569-575

[2] Casolo, C., Dolfi, S., 1996. The diameter of a conjugacy class graph of finite groups. Bulletin of the London Mathematical Society. 28(2): 141148

[3] Moradipour, K., Sarmin, N. H., Erfanian, A. On graph associated to conjugacy classes of some metacyclic 2-groups. Journal of Basic and Applied Scientific Research, 2013. 3(1):898-902.

[4] Omer, S. M. S., Sarmin, N. H., Erfanian, A., Moradipour, K., 2013. The probability that an element of a group fixes a set and its graph related to conjugacy classes. Journal of Basic and Applied Scientific Research, 3(10): 369-380.

[5] Omer, S. M. S., Sarmin, N. H. and Erfanian, A., 2013. The probability that an element of a symmetric group fixes a set and its application in graph theory. World Applied Sciences Journal, 27(12): 1637-1642.

[6] Humphreys, J. F. A Course in Group Theory. USA: Oxford University Press. 1996.

[7] Fraleigh, J. B. A First Course in Abstract Algebra. 7th. ed. USA: Pearson Education. 2003

[8] Rotman, J. J. Advanced Modern Algebra. USA: Pearson Education. 2002.

[9] Xiong, B., and Zheng, Z. Graph Theory. Shanghai, China: East China Normal University Press. 2010.

[10] Wilson, R. J. Introduction to Graph Theory. USA: Academic Press. 1972.

[11] Bollobas, B. Modern Graph Theory. USA: Springer. 1998.

[12] Chartrand, G., Zhang, P. Introduction to Graph Theory. New York, USA: McGraw-Hill. 2005. 